Case Report

Oopen Access

CrossMark

\title{
Is BHCG a good tumor marker for aggressive uterine leiomyosarcoma? case report and literature review
}

\begin{abstract}
An elevation of BHCG has been documented only in rare case reports of mesenchymal tumors, however, it is usually found to be high in germ cell tumors. ${ }^{.}$BHCG production in patients with mesenchymal tumors has been discussed as a paraneoplastic phenomenon as well as associated with bad prognoses. ${ }^{2,3}$
\end{abstract}

Keywords: germ cell tumors, urinary symptoms, salpingo-oophorectomy, leiomyosarcoma
Volume II Issue 2 - 2020

\author{
Sarah Alrubaish,' Marie-Claude Renaud ${ }^{2}$ \\ 'Gynecology Oncology, King Fahad Specialist Hospital Dammam, \\ Kingdom of Saudi Arabia \\ ${ }^{2}$ Gynecology Oncology, Laval University, Canada
}

Correspondence: Sarah Alrubaish, Gynecology Oncology, King Fahad Specialist Hospital Dammam, Kingdom of Saudi Arabia, Tel+966555 I83337, Email Sararubaish@hotmail.com

Received: April 06, 2020 | Published: April 30, 2020

\section{Case report}

We are presenting a 55-year old with an unremarkable medical history. She presented to her local hospital after noticing an increase in abdominal girth for almost 6 months. She did not have any vaginal bleeding, abdominal pain, or any urinary symptoms. The patient was nullipara. Laboratory evaluations were all normal. Tumor markers requested for her were normal except BHCG, which was elevated at 566. A computed tomography (CT) scan of the abdomen revealed a large uterine mass of 12.81 by 8.7 by $17.2 \mathrm{~cm}$ (Figure 1). Her chest $\mathrm{x}$-ray showed a right lung nodule of around $1.2 \mathrm{~cm}$ (Figure 2), which ultimately had progressed.

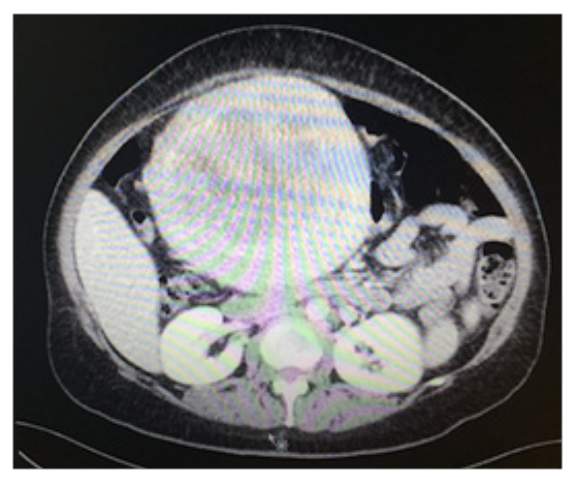

Figure I Axial computed tomography scan demonstrating a large uterine mass (arrow)

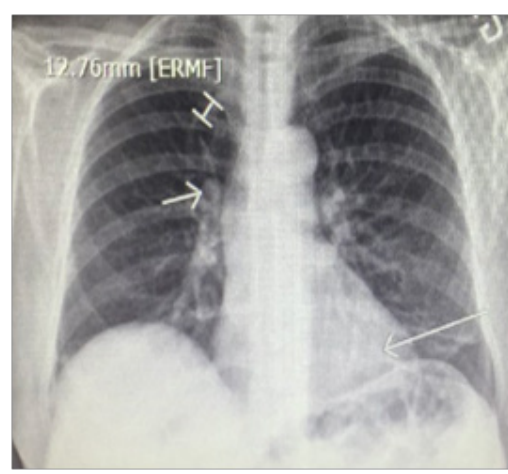

Figure 2 Chest $\mathrm{X}$-ray pre-operative with lung lesions (arrows).
The patient underwent a total abdominal hysterectomy and a bilateral salpingo-oophorectomy. An intra-operative frozen section revealed a uterine leiomyosarcoma. Final histopathology revealed a uterine leiomyosarcoma measuring $19 \mathrm{~cm}$, without lymphovascular invasion. Margins were clear of disease and the uterus was removed intact without morcellation. The remainder of the staging laparotomy (ovaries, nodes) were normal without metastasis, as expected.

The patient's day one post-operative BHCG levels went down to 27. She had an uneventful post-operative recovery. Two months post-surgery her BHCG levels started to progress to 74UI/L. Once the patient started chemotherapy and Gemcitabin-Docetaxol, her BHCG went down to $37 \mathrm{UI} / \mathrm{L}$ and normalized to $3 \mathrm{Ul} / \mathrm{L}$ during treatment.

The patient did well through the 4 cycles of chemotherapy. Although her BHCG was normalized, there was still documented metastatic disease in the thorax. She remained well with normal BHCG for three months after chemotherapy and then her BHCG started to rise again and progression of the disease was documented at the level of the chest (Figure 3).

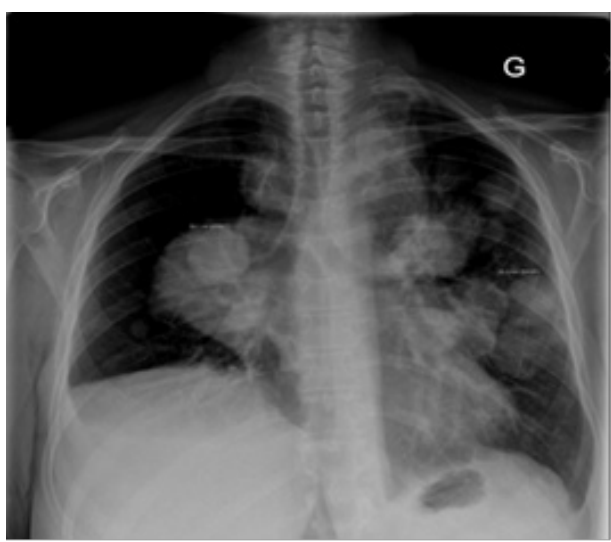

Figure 3 Chest $\mathrm{X}$-ray of the chest with disease progression after 3 months of chemotherapy.

The patient was offered a study protocol but while completing the randomization procedure she clinically deteriorated and finally was referred to palliative care. She died two months after disease progression was ascertained. 


\section{Discussion}

We are presenting an unusual case of paraneoplastic production of BHCG in an aggressive leiomyosarcoma in a postmenopausal woman. LMS is an aggressive and lethal disease, especially in an advanced stage and this is what our patient had. Is a high BHCG level an indicator of a poor prognosis? A review of case reports found that the majority of patients who were diagnosed with LMS regardless of the location of the tumor did not live more than one year, even with aggressive treatment. ${ }^{4,5}$

The production of BHCG has been described in the setting of an existing neoplasm in tumors of epithelial origin such as testicular embryonal tumors, epithelia ovarian cancer ${ }^{6,7}$ germ cell tumors, extra gonadal germ cell tumors, and, less commonly, lung carcinoma, hepatoma, and bladder cancer, but not in mesenchymal tissue. Therefore, patients with high BHCG produced by mesenchymal cells are considered to have paraneoplastic syndrome because almost every type of tumor has the potential to produce hormones or to induce cytokine and immunologic responses. ${ }^{8}$

In epithelial ovarian cancer a study in vitro has confirmed that HCG can induce the transformation of ovarian epithelial cells by increasing cell cycle progression and attenuating apoptosis to promote tumorigenic growth. ${ }^{6}$ Therefore, in epithelial ovarian cancer patients a study was conducted to investigate the association between the survival of patients with epithelial ovarian cancer and HCG and the result was negative. ${ }^{?}$

In gynecology, BHCG plays an important role in the diagnosis of pregnancy and pregnancy-related disorders. It has a diagnostic and therapeutic value, however, the alpha subunit is similar to FSH and $\mathrm{LH}$, and is therefore non-specific and not of use as a tumor marker. ${ }^{9}$

It has been documented that growth of human myometrial smooth muscles is stimulated by HCG, as a result of their having HCG receptors. So the question is whether the production of BHCG is secondary to high-grade mesenchymal cell proliferations and could be an indicator of the aggressiveness of the disease and could be used as a Tumor marker or if it is only a manifestation of a paraneoplastic syndrome. ${ }^{10}$ In the present case, we could extrapolate in view of the outcome, that the HCG acted more as a tumor marker mirroring disease evolution than as a marker for a paraneoplastic syndrome.

\section{Acknowledgments}

None.

\section{Conflicts of interest}

The authors declare there are no conflicts of interest.

\section{Funding}

None.

\section{References}

1. Morris CD, Hameed MR, Agaram NP, et al. Elevated $\beta$-hCG associated with aggressive Osteoblastoma. Skeletal Radiol. 2017;46(9):1187-1192.

2. Ou SM, Lee SS, Peng YJ, et al. Production of beta-HCG by spermatic cord leiomyosarcoma: A paraneoplastic syndrome? J Androl. 2006;27(5):643644.

3. Maryamchik E, Lyapichev KA, Halliday B, et al. Dedifferentiated Liposarcoma With Rhabdomyosarcomatous Differentiation Producing HCG: A Case Report of a Diagnostic Pitfall. Int J Surg Pathol. 2018;26(5):448-452.

4. Liang S, Stone G, Chalas E, et al. A high-grade uterine leiomyosarcoma with human chorionic gonadotropin production. Int J Gynecol Pathol. 2006;25(3):257-261.

5. Meredith RF, Wagman LD, Piper JA, et al. Beta-chain human chorionic gonadotropin-producing leiomyosarcoma of the small intestine. Cancer. 1986;58(1):131-135.

6. Guo X, Liu G, Schauer IG, et al. Overexpression of the $\beta$ subunit of human chorionic gonadotropin promotes the transformation of human ovarian epithelial cells and ovarian tumorigenesis. Am $J$ Pathol. 2011;179(3):1385-1393.

7. Ind T, Iles R, Shepherd J, et al. Serum concentrations of cancer antigen 125 , placental alkaline phosphatase, cancer-associated serum antigen and free beta human chorionic gonadotrophin as prognostic markers for epithelial ovarian cancer. Br J Obstet Gynaecol. 1997;104(9):1024-1029.

8. Viau M, Renaud MC, Grégoire J, et al. Paraneoplastic syndromes associated with gynecological cancers: A systematic review. Gynecol Oncol. 2017;146(3):661-671.

9. Leidinger B, Bielack S, Koehler G, et al. High level of beta-hCG simulating pregnancy in recurrent osteosarcoma: Case report and review of literature. J Cancer Res Clin Oncol. 2004;130(6):357-361.

10. Froehner M, Gaertner HJ, Manseck A, et al. Retroperitoneal leiomyosarcoma associated with an elevated $\beta-\mathrm{HCG}$ serum level mimicking extragonadal germ cell tumor. Sarcoma. 2000;4(4):179-181. 